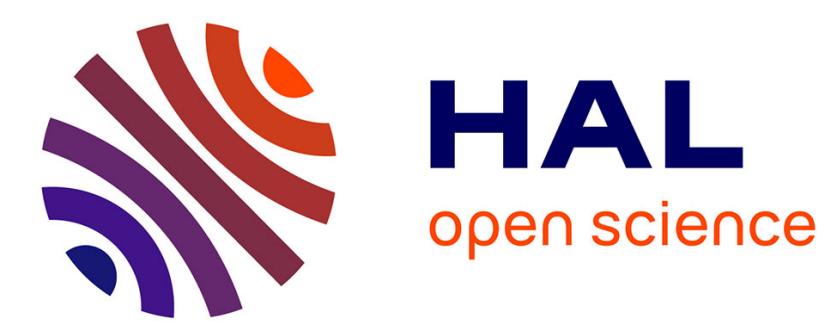

\title{
Phase Transitions in Inelastic Materials at Finite Strains: A Local Description
}

\author{
V. Levitas
}

\section{To cite this version:}

V. Levitas. Phase Transitions in Inelastic Materials at Finite Strains: A Local Description. Journal de Physique IV Proceedings, 1996, 06 (C1), pp.C1-55-C1-64. 10.1051/jp4:1996106 . jpa-00254137

\section{HAL Id: jpa-00254137 https://hal.science/jpa-00254137}

Submitted on 1 Jan 1996

HAL is a multi-disciplinary open access archive for the deposit and dissemination of scientific research documents, whether they are published or not. The documents may come from teaching and research institutions in France or abroad, or from public or private research centers.
L'archive ouverte pluridisciplinaire HAL, est destinée au dépôt et à la diffusion de documents scientifiques de niveau recherche, publiés ou non, émanant des établissements d'enseignement et de recherche français ou étrangers, des laboratoires publics ou privés. 


\title{
Phase Transitions in Inelastic Materials at Finite Strains: A Local Description
}

\author{
V.I. Levitas \\ Institut für Baumechanik und Numerische Mechanik, Universität Hannover, Appelstrasse 9A, \\ 30167 Hannover, Germany
}

\begin{abstract}
A general theory of phase transitions (PT) in the material point of simple inelastic materials with arbitrary constitutive equations is developed. The standard thermodynamical approach can be applied only after averaging of thermodynamical parameters, related to PT, over a PT duration. A PT criterion for the material point is derived, taking into account the temperature variation and dissipation due to $\mathrm{PT}$, as well as internal variables. Temperature variation in the course of PT is determined with the help of the entropy balance equation under the assumption that the process is adiabatic. Based on results for the material point, new nucleation and interface propagation criteria are derived. A postulate of realizability is applied to determine all unknown parameters in PT criteria. A model problem is solved.
\end{abstract}

\section{INTRODUCTION}

There are following known descriptions of martensitic PT: jump conditions for a moving interface, a variation of a volume fraction of phases in a multiphase material, a condition of nucleation in a finite volume and so on [1] - [3]. But a description of PT as a thermomechanical deformation process in a material point is lacking. This fact does not allow us to apply the standard methods of derivation of constitutive equations, as e.g. for a material point of elastoplastic materials. Moreover, an account for temperature variation in the course of $\mathrm{PT}$, temperature gradient and internal variables in the above approaches $[1]-[3]$ is nontrivial without a consequent theory for the material point. In the present paper $\mathrm{PT}$ is regarded as a thermomechanical inelastic deformation process, accompanied by change of all thermomechanical properties. The following important property is used: a norm (or components) of the transformation deformation gradient is fixed for each of crystallographically equivalent variants of PT and cannot be larger or smaller. All other configurations (crystal structures) with another norm of transformation deformation gradient are unstable and thus impossible. Based on these statements a general theory of $\mathrm{PT}$ in the material point of a simple inelastic body is developed. It is shown that a standard thermodynamical approach cannot be directly applied. It can be applied after averaging of thermodynamical parameters, related to PT, over a PT duration. A $\mathrm{PT}$ criterion is derived with account for the temperature variation due to $\mathrm{PT}$ and internal variables. It is shown that the temperature gradient does not contribute to PT criterion.

Nucleation and interface propagation conditions are derived, which generalize the conditions postulated in [3,4], for nonisothermal processes and media with internal variables. A model problem is solved. Some preliminary results were reported in [5].

Let a superscript $t$ denote transposition, dots mean contractions of the tensors. All statements are developed with respect to the reference configuration.

\section{THERMODYNAMICS OF MARTENSITIC PHASE TRANSITIONS IN A MATERIAL POINT}

Let us consider an uniformly deformed material point in a process of martensitic PT. We shall 
consider simple materials only, i.e. the material's response in the given point is independent of thermomechanical parameters in other points. We assume a multiplicative decomposition of a total deformation gradient $\boldsymbol{F}$ into elastic $\boldsymbol{F}_{e}$, thermal $\boldsymbol{F}_{\theta}$, transformational $\boldsymbol{F}_{t}$ and plastic $\boldsymbol{F}_{p}$ parts [6], i.e.

$$
\boldsymbol{F}=\boldsymbol{F}_{\mathrm{e}} \cdot \boldsymbol{F}_{\theta} \cdot \boldsymbol{F}_{t} \cdot \boldsymbol{F}_{\boldsymbol{p}}
$$

We introduce the internal dimensionless parameter $\xi(0 \leq \xi \leq 1)$ which is related with $F_{t}$ and has the following properties: PT starts at $\xi=0$ and finishes at $\bar{\xi}=1$; when $\xi$ varies between 0 and 1 , the transformation deformation gradient grows from 0 till $\boldsymbol{F}_{t \max }$. During this process all the thermodynamical properties of the phase 1 vary into the properties of the phase 2 . It is possible to define

$$
\xi:=\operatorname{tr} \boldsymbol{F}_{t} / \operatorname{tr} \boldsymbol{F}_{t \max }, \quad \xi:=\left|\boldsymbol{F}_{t}\right| /\left|\boldsymbol{F}_{t \max }\right|,
$$

but a lot of other definitions are acceptable. The internal parameter $\xi$ plays a similar role for the consideration of the uniformly deformed material point, as a volume fraction of martensite for the averaged description of $\mathrm{PT}$.

Let us define the specific Helmholtz free energy

$$
\psi=\psi\left(\boldsymbol{F}_{\mathrm{e}}(\xi), \theta(\xi), \boldsymbol{F}_{p}(\xi), \boldsymbol{g}(\xi), \boldsymbol{F}_{t}(\xi), \xi\right)
$$

where $g$ is a set of internal variables. We admit the second law of thermodynamic in the form of the Clausius-Duhem inequality

$$
\mathcal{D}=P^{t}: \dot{\boldsymbol{F}}-\rho \dot{\psi}-\rho s \dot{\theta}-\frac{\nabla \theta}{\theta} \cdot h \geq 0 .
$$

Here $\mathcal{D}$ is the rate of dissipation per unit volume, $P$ the first Piola-Kirchgoff nonsymmetric stress tensor, $\rho$ mass density in the reference configuration at $\xi=0, s$ the entropy, $\nabla \theta$ the temperature gradient, $h$ the heat flux. Substituting the rate of free energy $\dot{\psi}$ and taking into account the multiplicative decomposition Eq. (1) and $\dot{\boldsymbol{F}}_{\theta}=\frac{\partial \boldsymbol{F}_{\theta}}{\partial \theta} \dot{\theta}+\frac{\partial \boldsymbol{F}_{\theta}}{\partial \xi} \dot{\xi}$ in Eq. (4) yield

$$
\begin{aligned}
\mathcal{D} & =\left(\boldsymbol{F}_{\theta} \cdot \boldsymbol{F}_{t} \cdot \boldsymbol{F}_{p} \cdot \boldsymbol{P}^{t}-\rho \frac{\partial \psi}{\partial \boldsymbol{F}_{e}^{t}}\right): \dot{\boldsymbol{F}}_{e}+\left(\boldsymbol{P}^{t}: \boldsymbol{F}_{e} \cdot \frac{\partial \boldsymbol{F}_{\theta}}{\partial \theta} \cdot \boldsymbol{F}_{t} \cdot \boldsymbol{F}_{p}-\rho \frac{\partial \psi}{\partial \theta}-\rho s\right) \dot{\theta}+ \\
& +\left(\boldsymbol{P}^{t}: \boldsymbol{F}_{e} \cdot \boldsymbol{F}_{\theta} \cdot \frac{\partial \boldsymbol{F}_{t}}{\partial \xi} \cdot \boldsymbol{F}_{p}+\boldsymbol{P}^{t}: \boldsymbol{F}_{e} \cdot \frac{\partial \boldsymbol{F}_{\theta}}{\partial \xi} \cdot \boldsymbol{F}_{t} \cdot \boldsymbol{F}_{p}-\rho \frac{\partial \psi}{\partial \boldsymbol{F}_{t}^{t}}: \frac{\partial \boldsymbol{F}_{t}}{\partial \xi}-\rho \frac{\partial \psi}{\partial \xi}\right) \dot{\xi}+ \\
& +\left(\boldsymbol{P}^{t} \cdot \boldsymbol{F}_{\boldsymbol{e}} \cdot \boldsymbol{F}_{\theta} \cdot \boldsymbol{F}_{t}-\rho \frac{\partial \psi}{\partial \boldsymbol{F}_{p}^{t}}\right): \dot{\boldsymbol{F}}_{p}-\frac{\nabla \theta}{\theta} \cdot \boldsymbol{h}-\rho \frac{\partial \psi}{\partial \boldsymbol{g}^{t}}: \dot{\boldsymbol{g}} \geq 0 .
\end{aligned}
$$

The possibility of permutation of tensors in a scalar product is used, $\boldsymbol{P}^{t}: \dot{\boldsymbol{F}}_{e} \cdot \boldsymbol{F}_{\theta} \cdot \boldsymbol{F}_{t} \cdot \boldsymbol{F}_{p}=$ $=\boldsymbol{F}_{\theta} \cdot \boldsymbol{F}_{t} \cdot \boldsymbol{F}_{\boldsymbol{p}} \cdot \boldsymbol{P}^{t}: \dot{\boldsymbol{F}}_{\boldsymbol{e}}=\boldsymbol{F}_{e}^{-1} \cdot \boldsymbol{F} \cdot \boldsymbol{P}^{t}: \dot{\boldsymbol{F}}_{e}$ (decomposition (1) is taken into account). The assumption that the rate of dissipation is independent of $\dot{F}_{e}$ and $\dot{\theta}$ results in the hyperelasticity law and the expression for entropy, as well as in the reduced dissipative inequality:

$$
\begin{aligned}
\boldsymbol{F}_{e}^{-1} \cdot \boldsymbol{F} \cdot \boldsymbol{P}^{t} & =\rho \frac{\partial \psi}{\partial \boldsymbol{F}_{e}^{t}} ; \quad s=-\frac{\partial \psi}{\partial \theta}+\frac{1}{\rho} \boldsymbol{P}^{t}: \boldsymbol{F}_{e} \cdot \frac{\partial \boldsymbol{F}_{\theta}}{\partial \theta} \cdot \boldsymbol{F}_{t} \cdot \boldsymbol{F}_{p} \\
\mathcal{D} & =\boldsymbol{X}_{p}: \dot{\boldsymbol{F}}_{p}+\boldsymbol{X}_{\boldsymbol{g}}: \dot{\boldsymbol{g}}+\boldsymbol{X}_{h} \cdot \boldsymbol{h}+X_{\xi} \dot{\xi} \geq 0
\end{aligned}
$$

where $\quad \boldsymbol{X}_{p}=\boldsymbol{P}^{t} \cdot \boldsymbol{F}_{e} \cdot \boldsymbol{F}_{\theta} \cdot \boldsymbol{F}_{t}-\rho \frac{\partial \psi}{\partial \boldsymbol{F}_{p}^{t}} ; \quad \boldsymbol{X}_{g}=-\rho \frac{\partial \psi}{\partial \boldsymbol{g}^{t}} ; \quad \boldsymbol{X}_{h}=-\frac{\boldsymbol{\nabla} \theta}{\theta}$

and $\quad X_{\xi}=\boldsymbol{P}^{t} \cdot \boldsymbol{F}_{\boldsymbol{e}}:\left(\frac{\partial \boldsymbol{F}_{\theta}}{\partial \xi} \cdot \boldsymbol{F}_{t}+\boldsymbol{F}_{\theta} \cdot \frac{\partial \boldsymbol{F}_{t}}{\partial \xi}\right) \cdot \boldsymbol{F}_{\boldsymbol{p}}-\rho \frac{\partial \psi}{\partial \boldsymbol{F}_{t}^{t}}: \frac{\partial \boldsymbol{F}_{t}}{\partial \xi}-\rho \frac{\partial \psi}{\partial \xi}$ 
are dissipative forces conjugated with dissipative rates $\dot{\boldsymbol{F}}_{p}, \dot{\boldsymbol{g}}, \boldsymbol{h}$ and $\dot{\xi}$ respectively. The simplest assumption that each rate depends on the conjugate force only leads to evolution equations

$$
\dot{\boldsymbol{F}}_{p}=\boldsymbol{f}_{p}\left(\boldsymbol{X}_{p}, \xi\right) ; \quad \dot{\boldsymbol{g}}=\boldsymbol{f}_{g}\left(\boldsymbol{X}_{g}, \xi\right) ; \quad \boldsymbol{h}=\boldsymbol{f}_{h}\left(\boldsymbol{X}_{h}, \xi\right) ; \quad \dot{\xi}=f_{\xi}\left(X_{\xi}, \xi\right) .
$$

Eq. $(11)_{1}$ is the flow rule, Eq. $(11)_{2}$ is the evolution equation for the internal variables, Eq. (11) 3 is the generalized Fourier law and Eq. $(11)_{4}$ is the kinetic equation for PT. We consider the last equation for the description of PT only. The account for mutual influence of all thermomechanical processes can be made in a standard way.

We assume that the condition $\dot{\xi}=0$ is valid at $X_{\xi}=0$ only. Then it is possible to describe the equilibrium PT $(\dot{\xi} \rightarrow 0)$ with the equation $X_{\xi}=0$. This is one scalar equation, and for each $\xi$ and $\theta(\xi)$ it is always possible to choose six components of the stress tensor $\boldsymbol{P}(\xi, \theta(\xi))$ to satisfy $X_{\xi}=0$. If the actual stress variation follows this dependence, then the phase equilibrium is possible for arbitrary $\xi$. But from the experiments it follows that the phase equilibrium is impossible at $0<\xi<1$, only at $\xi=0$ and $\xi=1$ we have the stable equilibrium (see Introduction). At $0<\xi<1$ a nonequilibrium process proceeds which requires energy and stress fluctuations.

This situation seems new to us for continuum thermodynamics. In this case the standard thermodynamical approach cannot be applied. It is necessary to average the thermodynamical parameters, related with PT, over the duration of $\mathrm{PT}$ in order to filter off these fluctuations. We introduce the averaged dissipation rate as a dissipation work during PT divided on the duration $t_{p}$ of PT

$$
\mathcal{D}_{\xi}:=\frac{1}{t_{p}} \int_{0}^{t_{p}} X_{\xi} \dot{\xi} d t=\frac{1}{t_{p}} \int_{0}^{1} X_{\xi} d \xi=\frac{X}{t_{p}}=X \dot{\chi}, \quad \text { where } \quad X:=\int_{0}^{1} X_{\xi} d \xi, \quad \dot{\chi}:=\frac{1}{t_{p}}
$$

are the averaged dissipative force and rate. The definition of dissipative rate is logical, because a variation of the parameter $\xi$ during the time $t_{p}$ is equal one. The dissipative force is defined as a conjugate variable in the expression for the rate of dissipation. For the macroscopically equilibrium process $(\mathrm{PT})$ the following equation is valid

$$
X=\int_{\boldsymbol{F}_{t 1}}^{\boldsymbol{F}_{\mathrm{t} 2}}\left(\boldsymbol{F}_{p} \cdot \boldsymbol{P}^{t} \cdot \boldsymbol{F}_{\mathrm{e}} \cdot \boldsymbol{F}_{\theta}-\rho \frac{\partial \psi}{\partial \boldsymbol{F}_{t}^{t}}\right): d \boldsymbol{F}_{t}+\int_{0}^{1}\left(\boldsymbol{F}_{t} \cdot \boldsymbol{F}_{p} \cdot \boldsymbol{P}^{t} \cdot \boldsymbol{F}_{e}: \frac{\partial \boldsymbol{F}_{\theta}}{\partial \xi}-\rho \frac{\partial \psi}{\partial \xi}\right) d \xi=0
$$

The indices 1 and 2 denote the values before and after PT. Eq. (13) is the PT criterion for PT without dissipation.

Since practically all martensitic transformations, even in elastic materials, are accompanied with a dissipation and a hysteresis, the PT criterion has the following form

$$
X=k \text {. }
$$

Here $k$ is an experimentally determined value of dissipation due to PT, which can depend on $\theta, \dot{\chi}$, $\boldsymbol{F}_{\boldsymbol{p}}, \boldsymbol{g}, \ldots$. At $X<k \mathrm{PT}$ is impossible.

Let us consider the alternative expressions for $X$. From Eqs. (4) and (7) it follows

$$
\begin{aligned}
& X_{\xi} \dot{\xi}=\mathcal{D}-\boldsymbol{X}_{p}: \dot{\boldsymbol{F}}_{p}-\boldsymbol{X}_{g}: \dot{\boldsymbol{g}}-\boldsymbol{X}_{h} \cdot \boldsymbol{h}=\boldsymbol{P}^{t}: \dot{\boldsymbol{F}}-\rho \dot{\psi}-\rho s \dot{\theta}-\boldsymbol{X}_{p}: \dot{\boldsymbol{F}}_{p}-\boldsymbol{X}_{g}: \dot{\boldsymbol{g}} \\
& X=\int_{\boldsymbol{F}_{1}}^{\boldsymbol{F}_{2}} \boldsymbol{P}^{t}: d \boldsymbol{F}-\rho\left(\psi_{2}-\psi_{1}\right)-\int_{\theta_{1}}^{\theta_{2}} \rho s d \theta-\int_{0}^{t_{p}}\left(\boldsymbol{X}_{p}: \dot{\boldsymbol{F}}_{p}+\boldsymbol{X}_{g}: \dot{\boldsymbol{g}}\right) d t=k .
\end{aligned}
$$

Taking into account the decomposition (1) and Eq. $(9)_{1}$ for $X_{p}$ we obtain

$$
\begin{aligned}
X & =\int_{\boldsymbol{F}_{1}}^{\boldsymbol{F}_{2}}\left(\boldsymbol{P}^{t}: d \boldsymbol{F}_{e} \cdot \boldsymbol{F}_{\theta} \cdot \boldsymbol{F}_{t} \cdot \boldsymbol{F}_{p}+\boldsymbol{P}^{t}: \boldsymbol{F}_{e} \cdot d \boldsymbol{F}_{\theta} \cdot \boldsymbol{F}_{t} \cdot \boldsymbol{F}_{p}+\boldsymbol{P}^{t}: \boldsymbol{F}_{e} \cdot \boldsymbol{F}_{\theta} \cdot d \boldsymbol{F}_{t} \cdot \boldsymbol{F}_{p}\right)- \\
& -\rho\left(\psi_{2}-\psi_{1}\right)-\int_{\theta_{1}}^{\theta_{2}} \rho s d \theta+\int_{\boldsymbol{F}_{p_{1}}}^{\boldsymbol{F}_{p 2}} \rho \frac{\partial \psi}{\partial \boldsymbol{F}_{p}^{t}}: d \boldsymbol{F}_{p}+\int_{\boldsymbol{g}_{1}}^{\boldsymbol{g}_{2}} \rho \frac{\partial \psi}{\partial \boldsymbol{g}^{t}}: d \boldsymbol{g}=k .
\end{aligned}
$$


The expressions (16) and (17) are sometimes more convenient for the analysis, than Eq. (13). At $\frac{\partial \psi}{\partial F_{p}}=0 \mathrm{Eq} .(17)$ is more suitable for application, because in this case the plastic strain $\boldsymbol{F}_{p}$ disappears completely. Eq. (16) shows that $X$ is the total dissipation increment minus plastic dissipation increment and the dissipation increment due to the internal variables. Eq. (16) is more appropriate, when the moving interface is considered and the Hadamard's compatibility condition for $F_{i}$ is taken into account (see Section 4 ).

For elastic materials without internal variables and for isothermal processes, at $k=0$ it follows from Eq. (16)

$$
\int_{\boldsymbol{F}_{1}}^{\boldsymbol{F}_{2}} \boldsymbol{\sigma}: d \boldsymbol{F}=\rho\left(\psi_{2}-\psi_{1}\right)
$$

If PT proceeds at constant stress $\boldsymbol{P}_{o}$, then $\boldsymbol{P}_{o}^{t}:\left(\boldsymbol{F}_{2}-\boldsymbol{F}_{1}\right)=\rho\left(\psi_{2}-\psi_{\mathbf{1}}\right)$. This is the Maxwell rule. Eq. (16) is a generalization of the Maxwell rule for dissipative materials, nonisothermal processes and for variable stresses.

At small strains $\boldsymbol{F}=\boldsymbol{I}+\boldsymbol{\varepsilon}, \quad \boldsymbol{F}_{e}=\boldsymbol{I}+\boldsymbol{\varepsilon}_{e}, \quad \boldsymbol{F}_{\theta}=\boldsymbol{I}+\boldsymbol{\varepsilon}_{\theta}, \quad \boldsymbol{F}_{t}=\boldsymbol{I}+\boldsymbol{\varepsilon}_{t}, \quad \boldsymbol{F}_{p}=\boldsymbol{I}+\boldsymbol{\varepsilon}_{p}$, where $\varepsilon<<I, \varepsilon_{e}<<I, \varepsilon_{\theta}<<I, \varepsilon_{t}<<I, \varepsilon_{p}<<I$ are the corresponding strain tensors, $I$ is a second order identity tensor, $P=\sigma, P^{t}: \dot{\boldsymbol{F}}=\boldsymbol{\sigma}: \dot{\boldsymbol{\varepsilon}}$, where $\boldsymbol{\sigma}$ is a stress tensor, reference and actual configurations coincide and Eqs. (1), (6), $(9)_{1},(10),(16)$ and (17) take the form

$$
\begin{gathered}
\boldsymbol{\varepsilon}=\varepsilon_{e}+\boldsymbol{\varepsilon}_{p}+\boldsymbol{\varepsilon}_{\theta}+\boldsymbol{\varepsilon}_{t} ; \\
\boldsymbol{\sigma}=\rho \frac{\partial \psi}{\partial \boldsymbol{\varepsilon}_{e}} ; \quad s=\frac{\boldsymbol{\sigma}}{\rho}: \frac{\partial \boldsymbol{\varepsilon}_{\theta}}{\partial \theta}-\frac{\partial \psi}{\partial \theta} ; \\
\boldsymbol{X}_{p}=\boldsymbol{\sigma}-\rho \frac{\partial \psi}{\partial \boldsymbol{\varepsilon}_{p}} ; \quad X_{\xi}=\boldsymbol{\sigma}: \frac{\partial\left(\varepsilon_{t}+\boldsymbol{\varepsilon}_{\theta}\right)}{\partial \xi}-\rho \frac{\partial \psi}{\partial \boldsymbol{\varepsilon}_{t}}: \frac{\partial \varepsilon_{t}}{\partial \xi}-\rho \frac{\partial \psi}{\partial \xi} \\
X=\int_{\boldsymbol{\varepsilon}_{1}}^{\boldsymbol{\sigma}}: d \boldsymbol{\varepsilon}-\rho\left(\psi_{2}-\psi_{1}\right)-\int_{\theta_{1}}^{\boldsymbol{\varepsilon}_{2}} \rho s d \theta-\int_{0}^{t_{p}}\left(\boldsymbol{X}_{p}: \dot{\boldsymbol{F}}_{p}+\boldsymbol{X}_{g}: \dot{\boldsymbol{g}}\right) d t=k \\
\boldsymbol{\varepsilon}_{2} \boldsymbol{\sigma}: d\left(\boldsymbol{\varepsilon}_{e}+\boldsymbol{\varepsilon}_{\theta}+\boldsymbol{\varepsilon}_{t}\right)-\rho\left(\psi_{2}-\psi_{1}\right)-\int_{\theta_{1}}^{\theta_{2}} \rho s d \theta+\int_{\boldsymbol{g}_{1}}^{\theta_{1}} \rho \frac{\partial \psi}{\partial \boldsymbol{g}^{t}}: d \boldsymbol{g}+\int_{\boldsymbol{\varepsilon}_{p 1}}^{\boldsymbol{g}_{2}} \rho \frac{\partial \psi}{\partial \varepsilon_{p}}: d \boldsymbol{\varepsilon}_{p}=k
\end{gathered}
$$

Assume that both phases have the same elasticity tensor $\boldsymbol{E}$ and

$$
\rho \psi_{i}=0,5 \varepsilon_{e i}: E: \varepsilon_{e i}+\rho \psi_{i}^{\theta}=\rho \psi_{i}^{e}+\rho \psi_{i}^{\theta}, \quad i=1,2,
$$

where $\psi_{i}^{e}$ and $\psi_{i}^{\theta}$ are the elastic and thermal parts of free energy. Since

$$
\int_{\varepsilon_{e 1}}^{\varepsilon_{e 2}} \boldsymbol{\sigma}: d \varepsilon_{e}=\boldsymbol{E} \cdots \int_{\varepsilon_{e 1}}^{\varepsilon_{e 2}} \varepsilon_{e} d \varepsilon_{e}=0,5 \varepsilon_{e 2}: \boldsymbol{E}: \varepsilon_{e 2}-0,5 \varepsilon_{e 1}: \boldsymbol{E}: \varepsilon_{e 1}=\rho\left(\psi_{2}^{e}-\psi_{1}^{e}\right)
$$

it follows then from Eq. (23)

$$
X=\int_{\boldsymbol{\varepsilon}_{1}}^{\boldsymbol{\varepsilon}_{2}} \boldsymbol{\sigma}: d\left(\boldsymbol{\varepsilon}_{\theta}+\boldsymbol{\varepsilon}_{t}\right)-\rho\left(\psi_{2}^{\theta}-\psi_{1}^{\theta}\right)-\int_{\theta_{1}}^{\theta_{2}} \rho \dot{s} d \theta+\int_{\boldsymbol{g}_{1}}^{\boldsymbol{g}_{2}} \rho \frac{\partial \psi}{\partial \boldsymbol{g}^{t}}: d \boldsymbol{g}+\int_{\boldsymbol{\varepsilon}_{p 1}}^{\boldsymbol{\varepsilon}_{p 2}} \rho \frac{\partial \psi}{\partial \boldsymbol{\varepsilon}_{p}}: d \boldsymbol{\varepsilon}_{p}=k
$$


Since $\varepsilon_{\theta}=\alpha\left(\theta-\theta_{o}\right)$, where $\alpha=\alpha\left(\xi, \theta-\theta_{o}\right)$ is the thermal expansion tensor and $\theta_{o}$ is the reference temperature, we shall assume that for isothermal processes $\theta=\theta_{o}$ and we obtain $\varepsilon_{\theta}=0$. Consequently, for $\frac{\partial \psi}{\partial \boldsymbol{\varepsilon}_{p}}=\frac{\partial \psi}{\partial \boldsymbol{g}}=0$ and isothermal processes we have

$$
X=\int_{\boldsymbol{\varepsilon}_{t 1}}^{\boldsymbol{\varepsilon}_{\mathrm{t} 2}} \boldsymbol{\sigma}: d \boldsymbol{\varepsilon}_{t}-\rho\left(\psi_{2}^{\theta}-\psi_{1}^{\theta}\right)=k .
$$

There is one general problem: for calculation of integrals in Eq. (16) it is necessary to determine the stress variation (as well as $\boldsymbol{F}_{p}, \boldsymbol{g}, \theta$ ) during PT (variation of $\boldsymbol{F}_{t}$ ).

\section{DETERMINATION OF TEMPERATURE}

For the determination of a temperature variation in the course of PT we can use an entropy balance equation

$$
\rho \theta \dot{s}=\mathcal{D}+\operatorname{div} \boldsymbol{h}+\frac{\nabla \theta}{\theta} \cdot \boldsymbol{h} .
$$

Substitution of expression (7) for the rate of dissipation $\mathcal{D}$ in Eq. (28) yields

$$
\rho \theta \dot{s}=\operatorname{div} \boldsymbol{h}+\boldsymbol{X}_{p}: \dot{\boldsymbol{F}}_{p}+\boldsymbol{X}_{g}: \dot{\boldsymbol{g}}+X_{\xi} \dot{\xi} .
$$

According to Eq. $(6)_{2} s=s\left(\theta, \boldsymbol{P}, \boldsymbol{g}, \boldsymbol{F}_{p}, \boldsymbol{F}_{t}, \xi\right)$. Substitution of this expression and Eq. (11) 3 for the heat flux into Eq. (29) leads to the temperature evolution equation

$$
\begin{aligned}
\rho \nu \dot{\theta} & =\operatorname{div} f_{h}\left(-\frac{\nabla \theta}{\theta}\right)+\left(X_{p}-\rho \frac{\partial s}{\partial \boldsymbol{F}_{p}^{t}} \theta\right): \dot{\boldsymbol{F}}_{p}+\left(\boldsymbol{X}_{g}-\rho \frac{\partial s}{\partial \boldsymbol{g}} \theta\right): \dot{\boldsymbol{g}}+ \\
& +\left(X_{\xi}-\rho \frac{\partial s}{\partial \xi} \theta-\rho \frac{\partial s}{\partial \boldsymbol{F}_{t}^{t}}: \frac{\partial \boldsymbol{F}_{t}}{\partial \xi}\right) \dot{\xi}-\theta \frac{\partial s}{\partial \boldsymbol{P}^{t}}: \dot{\boldsymbol{P}}, \quad \nu:=\theta \frac{\partial s}{\partial \theta}
\end{aligned}
$$

where $\nu$ is the specific heat. Due to the divergence term the temperature in the given point can be defined after formulation and solution of a boundary-value problem. Taking into account a very short duration of PT $\left(10^{-6}-10^{-11} \mathrm{~s}\right)$, the adiabatic process can be assumed (similar to the processes in shock waves). In this case div $\boldsymbol{h}=0$ and Eq. (30) determines the temperature evolution in each material point independently, i.e. without solution of a boundary-value problem.

A general scheme of application of PT criterion (16) and temperature evolution equation (30) is following. All material properties, constitutive equations (11) and transformation strain $\boldsymbol{F}_{t}(\xi)$ should be given as function of $\xi$. Then assume that at some stress $P_{s}$ and temperature $\theta_{s} \mathrm{PT}$ in the given point starts. Stress variation $P(\xi)$, as well as $\boldsymbol{F}_{p}(\xi), \boldsymbol{g}(\xi)$, are determined by solution of a boundary value-problem or using some simplified models (e.g. [2]). The temperature can be determined by solution of $\mathrm{Eq}$. (30). In the general case mechanical and thermal equations are coupled and should be considered together. After determination of all parameters for $0 \leq \xi \leq 1$ the criterion (14) allows us to determine (iteratively or explicitly) the temperature $\theta_{s}$ at the given $P_{s}$ or one of the components of the stress tensor $\boldsymbol{P}_{s}$ with the remaining five components of $P_{s}$ and the temperature $\theta_{s}$ given.

Let us consider some specifications and simplifications of Eq. (30) at small strain. Assume the validity of Eq. (24) for the free energy with equal elastic properties of both phases and the following explicit expression for a thermal part of free energy [7]

$$
\psi_{1}^{\theta}=\psi_{o i}-s_{o i}\left(\theta-\theta_{o}\right)-\nu_{i} \theta\left(\ln \theta / \theta_{o}-1\right)-\nu_{i} \theta_{o}, \quad i=1,2 .
$$

Here $\nu_{i}>0$ are the specific heats, $s_{o i}$ and $\psi_{o i}$ are the constants. If the thermal expansion coefficient is independent of $\theta$, then from Eq. $(20)_{2}$ it follows

$$
\rho s=\rho s_{\circ}(\xi)+\rho \nu(\xi) \ln \frac{\theta}{\theta_{0}}+\sigma: \alpha(\xi) \quad \text { and } \quad \theta \frac{\partial s}{\partial \theta}=\nu(\xi),
$$


i.e. the accepted Eq. $(30)_{2}$ for the specific heat in a general nonlinear situation, coincides in this case with the specific heat in the expression for $\psi^{\theta}$. This is a reason for using a logarithmic term in Eq. (31).

Functions $s_{o}(\xi), \nu(\xi)$ and $\alpha(\xi)$ are usually unknown and we assume a linear approximation

$$
s_{o}=(1-\xi) s_{o 1}+\xi s_{o 2}, \quad \nu=(1-\xi) \nu_{1}+\xi \nu_{2}, \quad \alpha(\xi)=(1-\xi) \alpha_{1}+\xi \alpha_{2} .
$$

Then Eq. (30) can be transformed into the form

$$
\begin{aligned}
\rho \nu(\xi) \dot{\theta} & =\left(X_{\xi}-\rho \theta \Delta s_{o}-\rho \Delta \nu \theta \ln \theta / \theta_{o}-\sigma: \Delta \alpha \theta\right) \dot{\xi}- \\
& -\dot{\boldsymbol{\sigma}}: \boldsymbol{\alpha}(\xi) \theta+\boldsymbol{X}_{p}: \dot{\boldsymbol{\varepsilon}}_{p}+\boldsymbol{X}_{g}: \dot{\boldsymbol{g}},
\end{aligned}
$$

where $\Delta \boldsymbol{b}:=\boldsymbol{b}_{2}-\boldsymbol{b}_{1}$. If $\psi_{o}=(1-\xi) \psi_{o 1}+\xi \psi_{o 2}$ and $\psi_{o i}$ are independent of $\boldsymbol{g}$ and $\boldsymbol{\varepsilon}_{p}$, then $\boldsymbol{X}_{g}=0$ and $\boldsymbol{X}_{p}=\boldsymbol{\sigma}$. In this case Eq. (13) is the most convenient form of $X$. PT criterion results

$$
\begin{aligned}
\int_{\varepsilon_{t 1}}^{\varepsilon_{t 2}} \sigma: d \varepsilon_{t} & +\int_{0}^{1} \sigma: \Delta \boldsymbol{\alpha}\left(\theta-\theta_{o}\right) d \xi+\rho\left(\Delta s_{o}-\Delta \nu\right) \int_{0}^{1} \theta d \xi+ \\
& +\rho \Delta \nu \int_{0}^{1} \theta \ln \frac{\theta}{\theta_{o}} d \xi=k+\rho \Delta \psi_{o}+\rho\left(\Delta s_{o}-\Delta \nu\right) \theta_{o} .
\end{aligned}
$$

Eq. (35) shows that the thermal expansion tensors of each phase do not effect on PT criterion, only their difference contributes to the driving force. For an isothermal process at $\theta=\theta_{0}$ Eq. (35) coincides with Eq. (27) at $\left(\psi_{2}^{\theta}-\psi_{1}^{\theta}\right)=\Delta \psi_{0}$.

For isotropic $\boldsymbol{\alpha}=\alpha \boldsymbol{I}$ we have $\boldsymbol{\sigma}: \Delta \boldsymbol{\alpha}=3 \sigma_{o} \Delta \alpha, \quad \dot{\sigma}: \boldsymbol{\alpha}=3 \dot{\sigma}_{o} \alpha$, where $\sigma_{o}$ is a mean pressure and for fixed $\sigma_{o}$, for elastic materials and constant $X_{\xi}=k \mathrm{Eq}$. (34) is independent of the stress variation

$$
\rho\left((1-\xi) \nu_{1}+\xi \nu_{2}\right) \dot{\theta}=\left(k-\rho \theta \Delta s_{o}-\rho \Delta \nu \theta \ln \theta / \theta_{\circ}-3 \sigma_{\circ} \Delta \alpha \theta\right) \dot{\xi} .
$$

Choosing a proper $\theta_{o}$ we can meet the condition of smallness of $\left(\theta / \theta_{0}-1\right)$ and consequently $\ln \theta / \theta_{0} \simeq \theta / \theta_{o}-1$. In this case the temperature can be determined analytically, but the obtained equation is rather sophisticated for analysis. In the case $\Delta \nu=0$ we have

$$
d \theta=(A+B \theta) d \xi \quad \text { or } \quad \theta=\left(\frac{A}{B}+\theta_{s}\right) \exp (B \xi)-\frac{A}{B},
$$

where $A=\frac{1}{\rho \nu} k, B=-\frac{1}{\rho \nu}\left(\rho \Delta s_{o}+3 \sigma_{\circ} \Delta \alpha\right)$. PT criterion at $\Delta \nu=0$ reads as

$$
\int_{\varepsilon_{t 1}}^{\varepsilon_{t 2}} \sigma: d \varepsilon_{t}=\rho \nu B \int_{0}^{1} \theta d \xi+k+U
$$

where $U=\rho\left(\Delta \psi_{o}+\Delta s_{o} \theta_{o}\right)+3 \sigma_{o} \Delta \alpha \theta_{o}$, or taking into account Eq. (37)

$$
\int_{\varepsilon_{t 1}}^{\varepsilon_{t 2}} \sigma: d \varepsilon_{t}=\rho \nu\left(\frac{A}{B}+\theta_{s}\right)(\exp B-1)+U
$$

At small $B$ we have $\exp B \xi=1+B \xi$ and

$$
\theta=\theta_{s}+\left(A+B \theta_{s}\right) \xi ; \quad \int_{\varepsilon_{t_{1}}}^{\varepsilon_{t_{2}}} \sigma: d \varepsilon_{t}=\left(\rho \nu B \theta_{s}+k\right)(1+0.5 B)+U
$$


Simple analytical results can be obtained as well at variable pressure, if we assume $\alpha_{1}=\alpha_{2}=0$. In this case in Eqs. (35) and (36) $\Delta \alpha=0$, and at $\Delta \nu=0$ Eqs. (37) $-(40)$ are valid at

$$
A=k / \rho \nu, \quad B=-\Delta s_{o} / \nu, \quad A / B=-k / \rho \Delta s_{o}, \quad U=\rho\left(\Delta \psi_{o}+\Delta s_{o} \theta_{o}\right) .
$$

\section{CONDITIONS FOR NUCLEATION AND INTERFACE PROPAGATION}

Consider a volume $V$ of multiphase material with a boundary $S$. Let on one part of surface $S$ the stress vector $\boldsymbol{p}$ be prescribed and on the other part the velocity vector $v$ be given, but mixed boundary conditions are also possible. Assume that in some volume $V_{n}$ with the fixed boundary $\Sigma_{n}$, due to PT during the time $\Delta t$, the new nuclei appeared.

For each point of nuclei $V_{n} \mathrm{PT}$ criterion (16) should be meet. Integrating this criterion over the volume $V_{n}$ we obtain the necessary condition of nucleation

$$
\int_{V_{n}} X d V_{n}=\int_{V_{n}} k d V_{n_{0}},
$$

or in explicit form

$$
\begin{aligned}
\int_{V_{n}} \int_{F_{1}}^{F_{2}} P^{t}: d \boldsymbol{F} d V_{n} & =\int_{t}^{t+\Delta t} \int_{\Sigma_{n}} p \cdot v d \Sigma_{n} d t=\int_{V_{n}} \rho\left(\psi_{2}-\dot{\psi}_{1}\right) d V_{n}+\int_{V_{n}} \int_{\theta_{1}}^{\theta_{2}} \rho s d \theta d V_{n}+ \\
& +\int_{V_{n}} \int_{t}^{t+\Delta t}\left(\boldsymbol{X}_{p}: \dot{\boldsymbol{F}}_{p}+\boldsymbol{X}_{g}: \dot{\boldsymbol{g}}\right) d t d V_{n}+\int_{V_{n}} k d V_{n}
\end{aligned}
$$

where $v$ is the velocity on $\Sigma_{n}$ from the side of nucleus. Note that Gauss theorem was used. Eq. (43) is a necessary condition for nucleations, because from the point of view of a stable post-bifurcation deformation process in the whole volume $V_{n}$, at the same boundary data increment another process (e.g., nucleation in another volume, interface propagation and so on) can be more stable [8,9].

Assume that the volume $V_{n}$ is obtained by interface $\Sigma$ propagation with a normal velocity $v_{n}$ during time $\Delta t$, i.e. it is bounded by surfaces $\Sigma_{t}$ and $\Sigma_{t+\Delta t}$ at time $t$ and $t+\Delta t$ respectively, as well as by two lateral infinitesimal surfaces with the heights $v_{n} \Delta t$. Let us transform the stress work integral in Eq. (43).

On the moving coherent interface the position vector and the traction vector $\boldsymbol{p}$ are continuous, $p_{2}=p_{1}$, and due to compatibility condition $[6,8,9]$

$$
[\boldsymbol{F}]=-[\boldsymbol{v}] \boldsymbol{n} / v_{n}, \quad \text { whence } \quad[\boldsymbol{v}]=-[\boldsymbol{F}] \cdot \boldsymbol{n} v_{n} \quad \text { and } \quad[\boldsymbol{F}]=[\boldsymbol{F}] \cdot \boldsymbol{n} \boldsymbol{n} .
$$

Here $n$ is the unit normal to the interface, $[a]:=a_{2}-a_{1}$. Then neglecting all the terms of order $\Delta t^{2}$ we obtain

$$
\begin{aligned}
\int_{t}^{t+\Delta t} \int_{\Sigma} \boldsymbol{p} \cdot \boldsymbol{v} d \Sigma d t & =\left(\int_{\Sigma_{t+\Delta t}} \boldsymbol{p}_{1} \cdot \boldsymbol{v}_{1} d \Sigma_{t+\Delta t}-\int_{\Sigma_{t}} p_{2} \cdot \boldsymbol{v}_{2} d \Sigma_{t}\right) \Delta t=-\int_{\Sigma_{t}}[\boldsymbol{p} \cdot \boldsymbol{v}] \Delta t d \Sigma_{t}, \\
-[\boldsymbol{p} \cdot \boldsymbol{v}] & =-\boldsymbol{p} \cdot[\boldsymbol{v}]=\boldsymbol{n} \cdot \boldsymbol{P}^{t} \cdot[\boldsymbol{F}] \cdot \boldsymbol{n} v_{n}=\boldsymbol{P}^{t}:[\boldsymbol{F}] \cdot \boldsymbol{n} \boldsymbol{n} v_{n}=\boldsymbol{P}^{t}:[\boldsymbol{F}] v_{n},
\end{aligned}
$$

i.e. work-producing components of stress tensor are fixed in the course of PT. Substitution of Eq.

(45) into Eq. (43) with account for $d V_{n}=v_{n} \Delta t d \Sigma_{t}$ results in

$$
\begin{aligned}
& \int_{V_{n}} n \cdot \boldsymbol{P}^{t} \cdot[\boldsymbol{F}] \cdot \boldsymbol{n} d V_{n}=\int_{V_{n}} \boldsymbol{P}^{t}:[\boldsymbol{F}] d V_{n}=\int_{V_{n}} \rho[\psi] d V_{n}+\int_{V_{n}} \int_{\theta_{1}}^{\theta_{2}} \rho s d \theta d V_{n}+ \\
& +\int_{V_{n}} \int_{t}^{t+\Delta t}\left(\boldsymbol{X}_{p}: \dot{\boldsymbol{F}}_{p}+\boldsymbol{X}_{g}: \dot{\boldsymbol{g}}\right) d t d V_{n}+\int_{V_{n}} k d V_{n} \quad \text { and }
\end{aligned}
$$




$$
\boldsymbol{n} \cdot \boldsymbol{P}^{t} \cdot[\boldsymbol{F}] \cdot \boldsymbol{n}=\boldsymbol{P}^{t}:[\boldsymbol{F}]=\rho[\psi]+\int_{\theta_{1}}^{\theta_{2}} \rho s d \theta+\int_{t}^{t+\Delta t}\left(\boldsymbol{X}_{p}: \dot{\boldsymbol{F}}_{p}+\boldsymbol{X}_{g}: \dot{\boldsymbol{g}}\right) d t+k .
$$

Transition from an integral form (46) to a local one Eq. (47) is based on the assumption, that each point of interface can move independently of another point and the surface $\Sigma$ can be chosen arbitrary. If the interface can move without variation of its shape only (e.g. as a plane), i.e. the motion of interface points are not mutually independent, then only the integral form (46) is valid.

Eqs. (43) and (47) generalize equations, postulated in [2,3], for nonisothermal processes and media with internal variables. All simplifications from the Section 2, as well as temperature evolution equation are valid. Eq. (47) is valid at time $t+\Delta t$ as well $[2,3,8,9]$. Note that at transition from the material point to the finite volume or surface additional energy terms can appear and should be taken into account. E.g., a change of surface energy should be added to right side of Eqs. (43) and (47). Additional dissipation threshold $k_{\Sigma}$ due to intersection of interface with dislocations, point defects, grains and subgrains boundaries should be added to $k$ in Eq. (47).

Note, that Eqs. (43) and (47) are valid for noncoherent PT with small jump of displacement as well, since we are using the velocity vector $v$ from the side of nucleus, i.e. the rate of relative sliding along the interface $[\boldsymbol{v}]^{\boldsymbol{s}}$ is excluded from consideration and $[\boldsymbol{v}]$ should always meet the compatibility condition. We can define the total jump of velocity across the interface $[\boldsymbol{v}]^{T}=[\boldsymbol{v}]+[\boldsymbol{v}]^{s}$ and can use for the definition of $[\boldsymbol{v}]^{s}$ equations and extremum principles derived in $[8,9]$.

\section{THE POSTULATE OF REALIZABILITY}

PT criterion (42) (or its explicit forms (43), (46) and (47)) is only one scalar equation, which is not sufficient for the determination of all unknown parameters. To determine all unknown parameters $\boldsymbol{b}$, e.g. position, shape and orientation of nucleus, jump of $\boldsymbol{F}$, transformation $\boldsymbol{F}_{t}$ and plastic deformation $F_{p}$ gradients, related with $F_{p}$ temperature evolution, as well as functions $\boldsymbol{F}(\xi), \boldsymbol{F}_{t}(\xi), \boldsymbol{F}_{p}(\xi), \theta(\xi)$ and so on, let us use the postulate of realizability $[3,4,8,9]$.

Let for the given boundary conditions

$$
\int_{V_{n}^{*}} \boldsymbol{X}\left(\boldsymbol{b}^{*}\right) d V_{n}-\int_{V_{n}^{*}} k\left(\boldsymbol{b}^{*}\right) d V_{n}<0 \quad \forall \boldsymbol{b}^{*}
$$

for all possible PT parameters $b^{*}$, i.e. PT does not occur. If in the course of variation of boundary conditions the condition (42) is fulfilled the first time for some of parameters $b$, then PT will occur with this $\boldsymbol{b}$ (if condition (42) is not violated in the course of PT, e.g. interface propagation).

If, in the course of variation of boundary conditions the equality (42) is met for one or several $\boldsymbol{b}$, then for arbitrary other $\boldsymbol{b}^{*}$ inequality (48) should be held, as in the opposite case for this $\boldsymbol{b}^{*}$ condition (42) had to be met before it was satisfied for $\boldsymbol{b}$. Consequently, we obtain the extremum principle

$$
\int_{V_{n}} \boldsymbol{X}(\boldsymbol{b}) d V_{n}-\int_{V_{n}} k(\boldsymbol{b}) d V_{n}=0>\int_{V_{n}^{*}} \boldsymbol{X}\left(\boldsymbol{b}^{*}\right) d V_{n}-\int_{V_{n}^{*}} k\left(\boldsymbol{b}^{*}\right) d V_{n},
$$

for determination of all unknown parameters $b$. Explicit form of principle (49) looks as

$$
\begin{gathered}
\int_{V_{n}} \int_{\boldsymbol{F}_{1}}^{\boldsymbol{F}_{2}} \boldsymbol{P}^{t}: d \boldsymbol{F} d V_{n}-\int_{V_{n}} \rho\left(\psi_{2}-\psi_{1}\right) d V_{n}-\int_{V_{n}} \int_{\theta_{1}}^{\theta_{2}} \rho s d \theta d V_{n}- \\
\quad-\int_{V_{n}} \int_{t}^{t+\Delta t}\left(\boldsymbol{X}_{p}: \dot{\boldsymbol{F}}_{p}+\boldsymbol{X}_{g}: \dot{\boldsymbol{g}}\right) d t d V_{n}-\int_{V_{n}} k d V_{n}=0>
\end{gathered}
$$




$$
\begin{aligned}
\int_{V_{n}^{*}} \int_{\boldsymbol{F}_{1}^{*}}^{\boldsymbol{F}_{2}^{*}} P^{t *}: d \boldsymbol{F}^{*} d V_{n}-\int_{V_{n}^{*}} \rho\left(\psi_{2}^{*}-\psi_{1}^{*}\right) d V_{n}-\int_{V_{n}^{*}} \int_{\theta_{1}^{*}}^{\theta_{2}^{*}} \rho s^{*} d \theta^{*} d V_{n}- \\
-\int_{V_{n}^{*}} \int_{t}^{t+\Delta t}\left(\boldsymbol{X}_{p}^{*}: \dot{\boldsymbol{F}}_{p}^{*}+\boldsymbol{X}_{g}^{*}: \dot{\boldsymbol{g}}^{*}\right) d t d V_{n}-\int_{V_{n}^{*}} k^{*} d V_{n}
\end{aligned}
$$

for nucleation and as

$$
\begin{gathered}
\boldsymbol{P}^{t}:[\boldsymbol{F}]-\rho[\psi]-\int_{\theta_{1}}^{\theta_{2}} \rho s d \theta-\int_{t}^{t+\Delta t}\left(\boldsymbol{X}_{p}: \dot{\boldsymbol{F}}_{p}+\boldsymbol{X}_{g}: \dot{\boldsymbol{g}}\right) d t-k=0> \\
\boldsymbol{P}^{t *}:\left[\boldsymbol{F}^{*}\right]-\rho\left[\psi^{*}\right]-\int_{\theta_{1}^{*}}^{\theta_{2}^{*}} \rho s^{*} d \theta^{*}-\int_{t}^{t+\Delta t}\left(\boldsymbol{X}_{p}^{*}: \dot{\boldsymbol{F}}_{p}^{*}+\boldsymbol{X}_{g}^{*}: \dot{\boldsymbol{g}}^{*}\right) d t-k^{*}
\end{gathered}
$$

for interface propagation. In the particular case of nondissipative materials and isothermal processes, at small strains and equal elastic moduli, when PT occurs at constant stresses, the inequality (50) is equivalent to the Patel and Cohen condition $\sigma: \varepsilon_{t}>\sigma: \varepsilon_{t}^{*}[10]$.

\section{MODEL PROBLEM}

Let us consider the nucleation of a spherical inclusion with the radius $R$ in an infinite elasto-perfectly plastic body under applied external pressure $p$. Elastic properties of parent and new phases are the same, $\alpha_{1}=\alpha_{2}=0$. A similar problem is considered e.g. in [2], but our results will differ due to the new PT criterion and by taking into account the temperature variation in the nucleus.

Let $\varepsilon_{t}=\varepsilon_{o} I$. Then the pressure $\tilde{p}$ in a nucleus is determined by formulas [2]:

$$
\begin{array}{llll}
\text { in } & \text { elastic region } & \tilde{p}_{e} & =p-\frac{\varepsilon_{0}}{C}, \quad \varepsilon_{o} \leq \varepsilon_{0}^{\prime}:=\frac{2}{3} \sigma_{Y} C ; \\
& \text { in plastic region } & \tilde{p}_{p}=p-\frac{2}{3} \sigma_{Y}\left(\ln \frac{3 \varepsilon_{0}}{2 \sigma_{Y} C}+1\right), & \varepsilon_{0}>\varepsilon_{0}^{\prime} .
\end{array}
$$

Here $C=\frac{3(1-\nu)}{2 E}, E$ is Young's modulus, $\nu$ is Poisson's ratio, $\sigma_{Y}$ is the yield stress of parent phase, $\varepsilon_{0}^{\prime}$ is the strain corresponding to the onset of plastic flow in the parent phase. Note that $\sigma_{Y}$ can be positive or negative and due to the condition $\frac{3 \varepsilon_{0}}{2 \sigma_{Y} C}>0$ the signs of $\sigma_{Y}$ and $\varepsilon_{0}$ coincide.

Eqs. (37) - (39) for temperature variation and the PT criterion are valid with the constants defined by Eq. (41). Let us calculate the work integral

$$
\begin{aligned}
\int_{\varepsilon_{t 1}}^{\varepsilon_{t 2}} \boldsymbol{\sigma}: d \varepsilon_{t} & =3 \int_{0}^{\varepsilon_{o}} \tilde{p} d \varepsilon_{o}=3 \int_{0}^{\varepsilon_{o}^{\prime}} \tilde{p}_{e} d \varepsilon_{o}+3 \int_{\tilde{\varepsilon}_{o}^{\prime}}^{\varepsilon_{o}} \tilde{p}_{p} d \varepsilon_{o}= \\
& =3 p \varepsilon_{o}-\frac{2}{3} \sigma_{Y}^{2} C-2 \sigma_{Y} \varepsilon_{o} \ln \frac{3 \varepsilon_{o}}{2 \sigma_{Y} C} .
\end{aligned}
$$

Substituting Eq.(54) in Eq. (39) we obtain the PT pressure

$$
p=\frac{2}{3} \sigma_{Y}\left(\frac{\sigma_{Y} C}{3 \varepsilon_{o}}+\ln \frac{3 \varepsilon_{o}}{\sigma_{Y} C}\right)+\frac{\rho \nu}{3 \varepsilon_{o}}\left(\frac{A}{B}+\theta_{s}\right)(\exp B-1)+\frac{U}{3 \varepsilon_{o}} .
$$

For the description of PT progress we can consider the appearance of a new martensitic nucleus in a austenite + martensite mixture. In the first approximation, neglecting the energy of internal stresses 
in a mixture and assuming $\sigma_{Y}=c \sigma_{Y M}+(1-c) \sigma_{Y A}$, where $c$ is a volume fraction of martensite, $\sigma_{Y A}$ and $\sigma_{Y M}$ are the yield stresses of austenite and martensite, we can use Eq. (55). As for steels $\sigma_{Y M}$ is several times of $\sigma_{Y A}$, then PT pressure should be increased significantly for the increase of the martensite fraction. At $p \equiv 0 \mathrm{Eq}$. (55) describes temperature $\theta_{s}$ variation for the reaching of volume fraction $c$. As $\sigma_{Y}$ increases in several times with growth of $c$, after some $c$ the temperature $\theta_{s}$, determined from Eq. (55), can be less than $O K$. This means that some residual austenite can not be transformed into martensite due to cooling, which is in agreement with experiments for steels.

If we take into account significant increase of $\sigma_{Y A}$ and $\sigma_{Y M}$ with the temperature decrease [10], then at some $c \mathrm{Eq}$. (55) can have no solution with respect to $\theta_{s}$, while at decreasing $\theta_{s}$ the increase of $\Delta \psi^{\theta}$ is less than increase of resistance force, related with $\sigma_{Y}$. This means that PT is impossible at temperature, less than some critical one, which is also in agreement with experiments. A similar way of explanation for the existence of martensite finish temperature, but without using the explicit expression for PT criterion (e.g. Eq. (55)), was reported in [11].

\section{CONCLUDING REMARKS}

A developed finite strain theory of PT in the material point of inelastic continuum allows us a consistent derivation of nucleation and interface propagation conditions for rather general situation and arbitrary inelastic materials. Thereby, the temperature variation plays a very important role. When we neglect the temperature variation, in a problem considering PT in a thin layer under prescribed normal pressure and tangential displacement $[4,12]$, PT occurs in an infinitesimal thin layer, and plastic shear tends to infinity. Taking into the temperature variation due to PT results in a finite width of a layer and plastic shear [12]. Note that a simplified method of taking into account the effect of a temperature increase an PT condition is described in [2]. At the next step the simplified models of transforming volume should be developed, e.g. macroscopically uniformly [3] or with constant strain gradient deformed volume [13], with account for inertia effects $[6,13]$, with a wave mechanism of nucleus growth and so on.

\section{Acknowledgments}

The financial support of Alexander von Humboldt Foundation, as well as discussions with Prof. Erwin Stein are gratefully acknowledged.

\section{References}

[ 1 ] Kaganova I.M. and Roitburd A. L., Sov. Phys. Solid. State 29 (1987) 800-804.

$2]$ Fischer F.D., Berveiller M., Tanaka K. and Oberaigner E., Arch. Appl. Mech. 64 (1994) 54-85.

3 ] Levitas V.I., Mech. Res. Commun. 22 (1995) 87-94.

4 ] Levitas V.I., Journal de Physique IV, Colloque C2, 5 (1995) 41-46.

[5] Levitas V.I., Thermodynamische phasenumwandlungstheorie und eine Ähnlichkeit zur Plastizitätstheorie. Institut für Baumech. und Numer. Mech., Uni. Hannover, Bericht 95/1, (1995) 45 p.

[6] Levitas V.I., Thermomechanics of phase transformations and inelastic deformations in microinhomogeneous materials. Naukova Dumka, Kiev (1992) 248 p.

[ 7 ] Huo Y. and Müller I., Continuum Mech. Thermodyn. 5 (1993) 163-204.

[ 8 ] Levitas V.I., Post-bifurcation behaviour in finite elastoplasticity. Applications to strain localization and hase transitions. Institut für Baumech. und Numer. Mech., Uni. Hannover, Bericht 92/5, (1992) $107 \mathrm{p}$.

[9] Levitas V.I., Int. J. Eng. Sci., Parts 1 and 2, 33 (1995) 921-971.

[10] Patel J.R. and Cohen M., Acta Metall. 1 (1953) 531-538.

[11] Estrin E.I., Some problems of martensitic transformations. In Phase Transformation of Martensitic Type, Ed. Nemoshkalenko V.V. Naukova Dumka, Kiev (1993) pp. 110-139.

[12] Levitas V.I., Mech. Res. Commun. (1995, submitted).

[13] Levitas V.I., Mech. Res. Commun. 21 (1994) 11-17. 